\title{
A Class of Evolutionary Algorithms for Determining Shortest Path Routing in 5G Ultra Dense Heterogeneous Networks
}

\author{
${ }^{1}$ Debashis Dev Misra \\ ${ }^{1}$ Royal School of \\ Engineering and \\ Technology, Royal Global \\ University, Guwahati, \\ Assam, India. \\ debashish.dm@gmail.com
}

\author{
${ }^{2}$ Kandarpa Kumar Sarma \\ ${ }^{2}$ Department of Electronics \\ and Communication \\ Engineering, Gauhati \\ University, Assam, India. \\ kandarpaks@gmail.com
}

\author{
${ }^{3}$ Pradyut Kumar Goswami \\ Assam Science and \\ Technology University, \\ Assam, India. \\ pradyutgoswami@yahoo.co \\ $\mathrm{m}$
}

\author{
${ }^{4}$ Utpal Bhattacharjee \\ ${ }^{4}$ Department of Computer \\ Science, \\ Rajiv Gandhi University, \\ Arunachal Pradesh, India. \\ utpal.bhattacharjee@rgu.ac.
}

\begin{abstract}
Ultra Dense Network (UDN), an important element of the upcoming $5 \mathrm{G}$ networks are characterised by extremely dynamic operations due to the presence and mobility of large number of users spread over small cells of varying sizes. It makes optimal path between the source/destination pairs for communication and data transmission be highly dynamic in nature and hence a challenging issue to deal with. Under such dynamic backdrops, routing procedures have to exhibit robustness, scalability and time efficiency in order to ensure seamless link reliability and Quality of Service (QOS) of the network. In the proposed work, the shortest optimal route of the source and destination pair is found using a combination of evolutionary optimization algorithms namely Genetic Algorithm (GA), Particle Swarm Optimization (PSO) Algorithm and our novel hybrid PSOGA approach which searches for an optimized solution by determining cost functions of individual fitness state and comparing states generated between individual solutions. Application of all the three above mentioned algorithms to the Shortest Path Routing Problem in UDNs and the results obtained have shown that the hybrid PSO-GA comparatively provided enhanced optimized solution.
\end{abstract}

Keywords- Ultra Dense Network, 5G, Routing, Shortest Path Routing Problem, Particle Swarm Optimization, Genetic Algorithm, UDN, SPP, GA, PSO.

\section{INTRODUCTION}

The communication industry is in a state of continuous evolution due to the dramatic and exponential growth of data traffic volume. This enormous growth can be attributed to the ever increasing demand for smart devices, mobile multimedia services like video conferencing, online gaming with High Definition (HD) and Ultra High Definition (UHD) Resolution video, etc. Many international forecasting agencies predict that there shall be a violent data storm and around 40 billion wireless connected IOT devices by 2025 . The upcoming 5G cellular networks shall usher in an era of over 1Gbps connectivity, around $1 \mathrm{mS}$ latency, over $50 \mathrm{MHz}$ bandwidth, lower cost per bit transmission etc. The feasible solutions to cope with these aggressive data growth rate are categorized into three categories, namely, broadening the available bandwidth, improving the spectral efficiency and its reuse and the network infrastructure densification. In our research and proposed work, we focus on the network densification criteria which are the most proven effective factor in the past. Densification gain comes from splitting of Macrocell into cells into varying sizes like Microcell, Picocell and Femtocell etc. as per user requirements thereby serving more user equipments (UEs) simultaneously.

In such a backdrop, Ultra Dense Network (UDN) is a new paradigm shift in the realization of the true potential of $5 \mathrm{G}$ cellular networks. In an UDN, the number of access/serving nodes will be strengthened and be either same to the or surpass the number of user equipments(UEs).Hence, UDNs are evolving as one of the core challenges and research areas of $5 \mathrm{G}$ cellular networks that would bring in far reaching modifications in future networks[1].

UDNs are defined as networks where the number of cells are more than active users [12].In other words,

$$
\lambda_{a}>>\lambda_{u}
$$

where $\boldsymbol{\lambda}_{\boldsymbol{a}}$ is the density of access points and $\boldsymbol{\lambda}_{\boldsymbol{u}}$ is the density of users.

The second definition of UDN was represented in terms of the cell density. In [13] Ding et al. provided a quantitative measure of the density at which a network can be considered ultra-dense (> 1000 cells $/ \mathrm{km} 2$ ).The first definition converges with the second definition given that the density of active users considered in dense urban scenarios is upper bounded by about 600 active users/km2 [14].

The mobile end user clients would operate on a large number of sparsely deployed small cells and access nodes in their surroundings or in hotspot areas like airports, trains, metro/train stations, etc. Small cell networks synchronize with macro cells, either in the same spectrum or on a dedicated carrier channel.

Figure 1 shows two situations of the UDN. 


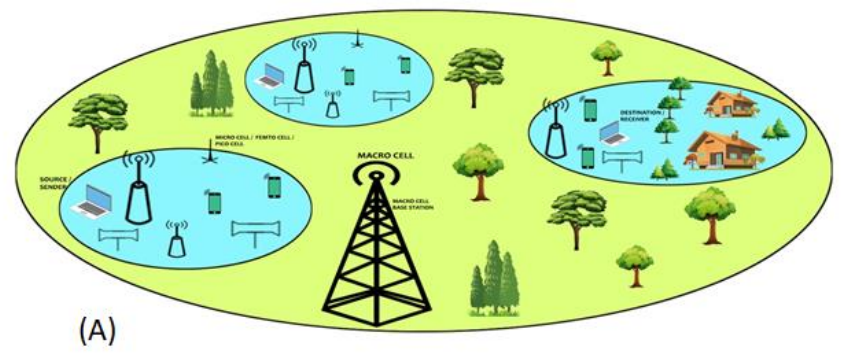

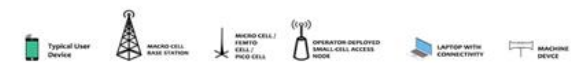

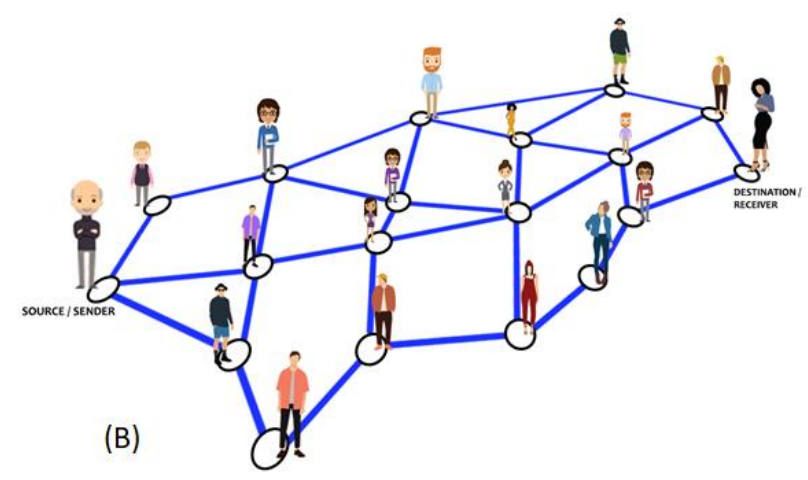

Fig.1. Two situations of UDN with mobile users / relay nodes as source/destination pairs and large numbers of small cells $r$ varying sizes co-existing with macro cells.

In Fig. 1 (A) a generic UDN with mobile users / relay nodes as source/destination pairs with deployment of large numbers of small cells of varying sizes including micro cell, femtocell, pico cell densify the network which co-exist and synchronize with the macro cells are shown. In Figure 1. (B), UDN with mobile users and user equipments (UEs) as source/ destination pair is shown. The source/destination path has a large number of intermediate users along the different cells. The routing path formation takes place in between the source and destination through these intermediate nodes.

The extremely dynamic nature of UDNs due to the mobility and presence of large number of users imply that the optimal path between the source/destination pairs for communication and data transmission will be highly fragile and dynamic in nature. Under such alternating backdrops, routing procedures and algorithms have to be designed to be robust and scalable in nature in order to ensure seamless connectivity and quality of service of the network. The shortest optimal route of the source/destination pair is found using a combination of evolutionary optimization algorithms namely Genetic Algorithm (GA), Particle Swarm Optimization (PSO) Algorithm and our proposed hybrid PSO-GA which searches for optimized solution by representing it as a Shortest Path Routing Problem (SPP).

In [2], [3], authors Kennedy and Eberhart developed and proposed the Particle Swarm Optimisation computational technique in 1995. PSO is motivated by the behaviour of social organisms/animals in groups, such as birds, ant colonies and fish schooling. It is a bio-inspired population-based optimization technique and part of evolutionary computing.PSO tries to find a feasible solution using a random population of particles. These particles or candidate solutions move through the n-dimensional search space of an optimization problem .The particles adjust their positions and velocities based on their own experience. And also based on the experience of neighbouring particles. This process continues iteratively until the particles finally converge to a feasible solution based on the assigned fitness value.

Genetic Algorithm is a random search and computational technique that is used to solve a wide array of Optimization and Search Problems.GA is an adaptive heuristic search algorithm and part of evolutionary computing or biologically inspired [7]. GAs is motivated by Darwin's theory of evolution "Survival of the fittest". It represents that theory among individuals of consecutive generations for solving an optimization problem. It reflects the theory of nature where the best or fittest individuals or chromosomes survive and come together for reproduction. They produce offspring's and pass the genes to the next generation which becomes even more competent and fit.

\section{RELATED WORK AND TERMINOLOGIES}

In [16], Ge et al. discusses the backhaul network capacity and energy efficiency to calculate densification limits to be applied for deployment in 5G UDNs. They point out that routing techniques will undergo major changes and modifications in $5 \mathrm{G}$ networks. Multihop relay optimization is going to be one of the critical challenges in the $5 \mathrm{G}$ ultra dense cellular networks. Both the backhaul and front haul traffic needs to be forwarded to the destination in the distributed network architecture. Relay small cell BS should be carefully selected and considered in $5 \mathrm{G}$ networks. In order to cope with the challenges of $5 \mathrm{G}$, design and development of new wireless multi-hop relay schemes and distributed routing algorithms are of outmost concern and importance.

In [17], Zhang et al. proposes cooperative routing mechanisms for $5 \mathrm{G}$ cellular networks. $\mathrm{CR}$ procedure is a cross layer routing procedure involving the physical and network layer. It utilizes routing path selection functions of network layer and the technology of cooperative transmission of the physical layer. The proposed work discusses a weighted power allocation scheme to maximize the network lifetime by considering various elements and parameters. The work proposes a novel cooperative routing procedure and algorithm for maximizing network lifetime on the basis of power allocation. 
Ahn et al. in [5] discuss and propose a modified Genetic Algorithm with emphasis on the population size of the candidate solutions. The population size equation is based on certain equations and criteria. It attempts to provide solutions to SP routing problem in wireless networks. Chromosomes with variable length along with their corresponding genes are being studied for formulating the SP problem. To maintain the diversity of the population, the crossover and mutation operator are used to a satisfactory extent. A simple repair function is also proposed. It aims to correct all sub-optimal or infeasible solutions or chromosomes. The genetic operators used provide optimal quality of solution and better rate of convergence. A desired quality solution is achieved through a population size equation. All the practical experiments and simulations performed show that the proposed algorithm provides better solution and higher rate of convergence than others.

In [18], Mohemmed et al. proposes a modified Particle Swarm Optimization (PSO) algorithm. This algorithm also attempts to provide solutions to Shortest Path Routing Problem in wireless networks. They try to avoid formation of loops in the constructed path for particle formation. The authors use a priority based encoding scheme for the same. They point out that the proposed PSO can find optimal solutions with higher accuracy and less computation time. They also say that the performance of the proposed PSO is better than all other proposed GAs for the SP problem.

\section{PROPOSED APPROACH}

\section{A. Proposed Pso for the Sp Problem in the Udn}

In our proposed Particle Swarm Optimization (PSO) search space, each particle of the initial random population has its own fitness value. The fitness value is based on the value returned by the objective function. The particles then move through the search space by following its current optimum values.

At the end of each iteration, the particles store the updated fitness value or feasible solution it has visited in its memory called the p-best or personal best value. The best fitness value obtained by particle to particle interaction is called the g-best or global best value. This process continues iteratively until a favourable solution or convergence is achieved.

At the end of each iteration, the movement of the particle is computed by the following equation

$$
x_{i}(\mathrm{t}+1) \leftarrow x_{i}(\mathrm{t})+v_{i}(\mathrm{t})
$$

$$
\begin{aligned}
& v_{i}(\mathrm{t}+1) \leftarrow \omega v_{i}(\mathrm{t})+c_{1} r_{1}\left(\operatorname{pbest}_{i}(\mathrm{t})-x_{i}(\mathrm{t})\right)+c_{2} r_{2}(\operatorname{gbest}(\mathrm{t})- \\
& \left.x_{i}(\mathrm{t})\right)
\end{aligned}
$$

Equations (2) denotes that the position of particle $i$ at time $\mathrm{t}$ is denoted by $x_{i}(\mathrm{t})$. Equations (3) denotes that the velocity of particle $i$ at time $\mathrm{t}$ is denoted by $v_{i}(\mathrm{t})$. The current best position found by the particle itself so far is given by pbest $_{i}(\mathrm{t})$.And the best position found by the whole swarm so far is denoted by gbest $(\mathrm{t}) . \omega$ is an inertia weight scaling the previous time step velocity. The two acceleration coefficients c1 and c2 scale the influence of the best personal position of the particle $\left(\right.$ pbest $\left._{i}(\mathrm{t})\right)$ and the global best position (gbest $(\mathrm{t})$ ). r1 and $\mathrm{r} 2$ are random variables within the range of 0 and 1.

Equations (4) and (5) define how the personal and global best position values are updated at time $t$. It is assumed below that the swarm consists of s particles. The objective function $\mathrm{f}$ is used to calculate the fitness of the particles with a minimal task.

Thus, $i \in 1 \ldots s$

pbest $_{i}(\mathrm{t}+1)=\left\{\right.$ pbest $_{i}(\mathrm{t})$ if $\mathrm{f}\left(\right.$ pbest $\left._{i}(\mathrm{t})\right) \leq \mathrm{f}\left(x_{i}(\mathrm{t}+1)\right)$

$\left\{x_{i}(\mathrm{t}+1)\right)$ if $\mathrm{f}\left(\right.$ pbest $\left._{i}(\mathrm{t})\right) \leq \mathrm{f}\left(x_{i}(\mathrm{t}+1)\right) \quad \ldots .$.

$\operatorname{gbest}(\mathrm{t}+1)=\min \{\mathrm{f}(\mathrm{y}), \mathrm{f}(\operatorname{gbest}(\mathrm{t}))\}$

where, $\mathrm{y} \in\left\{\right.$ pbest $_{0}(\mathrm{t})$, pbest $_{1}(\mathrm{t}), \ldots . .$, pbest $\left._{s}(\mathrm{t})\right\}$

The process flow for the PSO Algorithm is shown below.

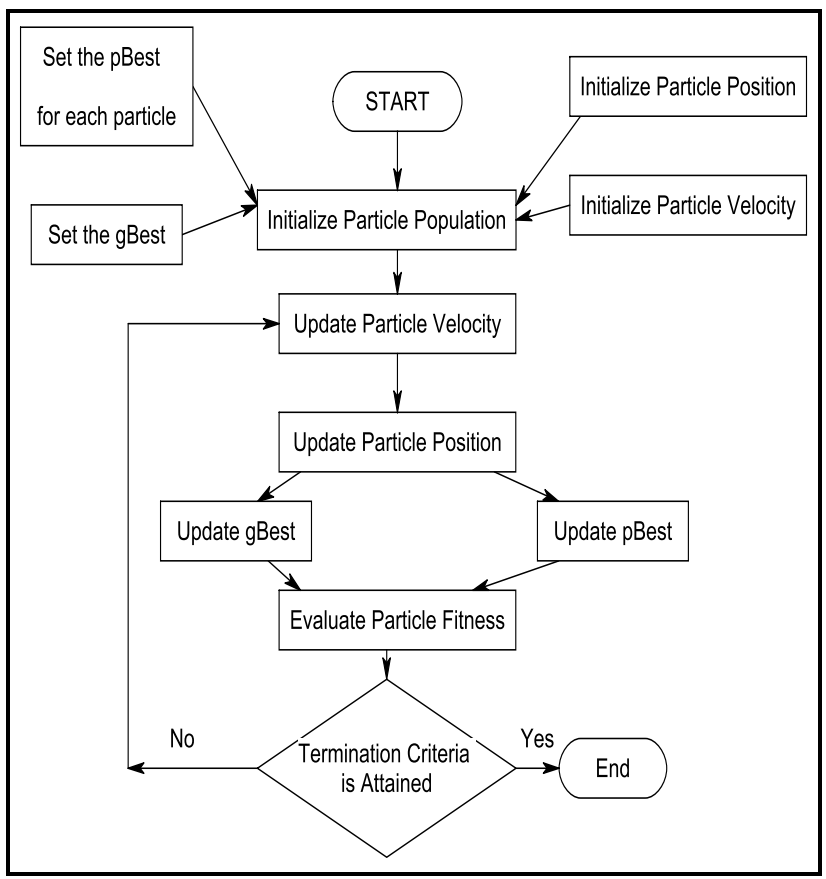

Fig. 2. Process of PSO Algorithm 
In general, the termination condition mentioned in the algorithm flowchart depends on the type of problem being applied. In our case, the condition is true when most of the particles (feasible solutions) converge to an optimal solution and matches with the solution provided by Dijkstra's Algorithm.

\section{B. Proposed Ga for the Sp Problem in the Udn}

The Genetic Algorithm proposed in our work, consists of an initial population of either feasible or infeasible solutions also known as chromosomes. The chromosomes or solutions are of variable length to increase the accuracy and efficiency of the algorithm.

The fundamental topology of Ultra Dense Networks can be represented by the directed graph $\mathrm{G}=(\mathrm{N}, \mathrm{A})$ where $\mathrm{N}$ is the set of nodes (vertices). A is the set of its links (edges or arcs) [4-6]. There is a cost $C_{i j}$ associated with each link (i, j). The costs are specified by the cost matrix $\mathrm{C}=\left[C_{i j}\right]$ where $C_{i j}$ denotes a cost of transmission of a data packet on link $(i, j)$. Source and destination nodes or relays are denoted by $\mathrm{S}$ and $\mathrm{D}$.

Each link has the link connection indicator denoted by $I_{i j}$, which plays the role of a chromosome map and provides information on whether the link from node $i$ to node $j$ is included in a routing path or not.

It can be defined as follows:

$I_{i j}=\{1$ if the link from node $\mathrm{i}$ to node $\mathrm{j}$ exists in the routing path

$=0$ otherwise.

The above definition of Equation 6 has led us to formulate the Shortest Path Routing Problem in an UDN as a combinatorial optimization problem with minimizing the objective function as:

Minimize

$$
\sum_{i=S}^{D} \sum_{\substack{j=s \\ j !=i}}^{D} C_{i j} \cdot I_{i j}
$$

Subject to

$$
\begin{aligned}
\sum_{\substack{j=s \\
j !=i}}^{D} I_{i j}-\sum_{\substack{j=s \\
j !=i}}^{D} I_{j i} & =1 \text { if } \mathrm{i}=\mathrm{s} \\
& =-1 \text { if } \mathrm{i}=\mathrm{D} \\
& =0 \text { otherwise }
\end{aligned}
$$

\section{A. Genetic Representation}

In our research work, the proposed Genetic Algorithm is represented as a chromosome. It consists of genes represented as positive integers. The integer values represent the ids of the nodes or relays through which the path passes. The gene of the first and last locus is always reserved for the source and destination nodes. The chromosome length varies for each feasible solution but it should not exceed $\mathrm{N}$, where $\mathrm{N}$ is the maximum number of nodes or relays in the network. The optimal routing path if formed based on the topological information (routing table) provided by real time routing protocols.

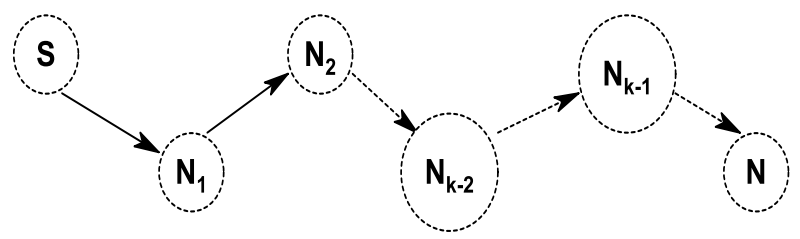

\begin{tabular}{|l|l|l|l|l|l|l|l|}
\hline Locus & 1 & 2 & 3 & $\cdots$ & L-2 & L-1 & L \\
\hline $\begin{array}{l}\text { Chromo } \\
\text { some }\end{array}$ & $\mathbf{S}$ & $N_{1}$ & $N_{2}$ & $\cdots \cdots$ & $N_{k-2}$ & $N_{k-1}$ & D \\
\hline
\end{tabular}

Fig.3. Diagram of a sample routing path and its encoding scheme.

Fig.3. displays a sample chromosome from node $\mathrm{S}$ to node D. The target chromosome is a list of nodes forming the routing path. In the above figure, $L$ represents the total number of nodes forming the path.

\section{B. Population Initialization}

Two relevant issues have been considered for population initialization of GAs, namely, the initial population size and the population initialization procedure [7], [8].In our practical experiments, the initial population size are equal to the number of nodes in the network. This has been done in order to have a fair comparison of the proposed algorithms.

From the literature, we have found that heuristic initialization and random initialization are the two standard procedures to generate the initial population. In our proposed work, we have used the random initialization procedure.

\section{Fitness Function}

The Shortest Path Problem (SPP) needs to find minimal cost path between the source and destination. Therefore the definition of the fitness function should be very fundamental in nature. The cost of a path indicated by the chromosome is used to calculate its fitness. The fitness function is denoted by: 


$$
f_{i}=\frac{1}{\sum_{j=1}^{L_{i}-1} C_{g_{i}(j), g_{i}(j+1)}}
$$

In equation (8), $f_{i}$ represents the fitness score of the $i$-th chromosome. The length of the $i$-th chromosome is denoted by $L_{i} \cdot g_{i}(j)$ denotes the gene or node of the $\mathrm{j}$-th locus in the $i$-th chromosome. $\mathrm{C}$ is the link cost between the nodes.

\section{Selection}

In selection, the key thought is to give the preference to the chromosomes with higher fitness scores. The selection or reproduction operator intends to improve the average quality of the population to get optimum solutions [7], [8]. After going through the related literature, we have employed pair wise tournament selection without replacement. Through this procedure, the fitter among the two chromosomes is picked up and chosen. The only constraint being the parent should not be represented by the same chromosome twice.

E. Crossover- Here, the present feasible solutions are studied and examined. The key idea is to generate better solutions from current ones [7], [8].In our proposed work, each partial route of two selected chromosomes is exchanged to produce an offspring such that it forms only one route. This is done by the crossover operator.

F. Mutation- Population diversity is maintained through this operator. This is done to prevent the premature convergence to a global solution. In order to do this, random genes are inserted in offspring over successive generations. This happens through actual change or flip of the genes in the candidate solutions or chromosomes. The process flow for the proposed GA is shown below

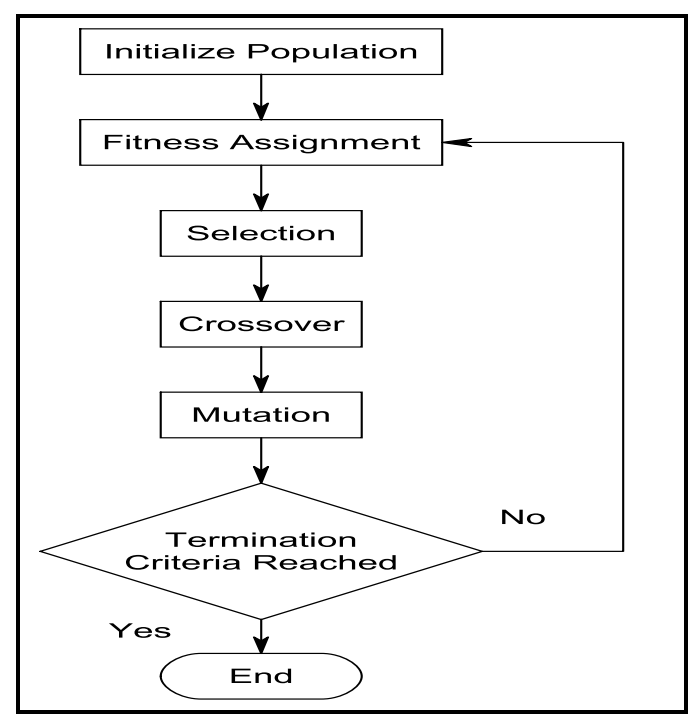

Fig.4. Process of Genetic Algorithm

\section{Proposed Hybrid Pso-ga for the Sp Problem in the $U d n$}

The performance analysis of the PSO and GA has been investigated and explored in several papers [2], [3], [10][11] . The investigation gives us the understanding that each one has its own advantages and disadvantages in the context of the problem being applied. The problem of stagnation of PSO has also been discussed. Also the comparison between both these algorithms have been formulated in [9], where the author stresses on the fact that PSO performs well in the early iterations but develops problems in reaching a near-optimal solution. The reviewed literature has led us to believe that in case of our proposed work as the size of the network increases or decreases in a dense network, the efficiency and accuracy of the computed results can be compromised to a certain extent. Under these circumstances we have moved forward and proposed a novel hybrid PSO-GA algorithm to provide a optimal solution to the SP problem in an dense network. The pseudo code for proposed hybrid PSO-GA has been shown below.

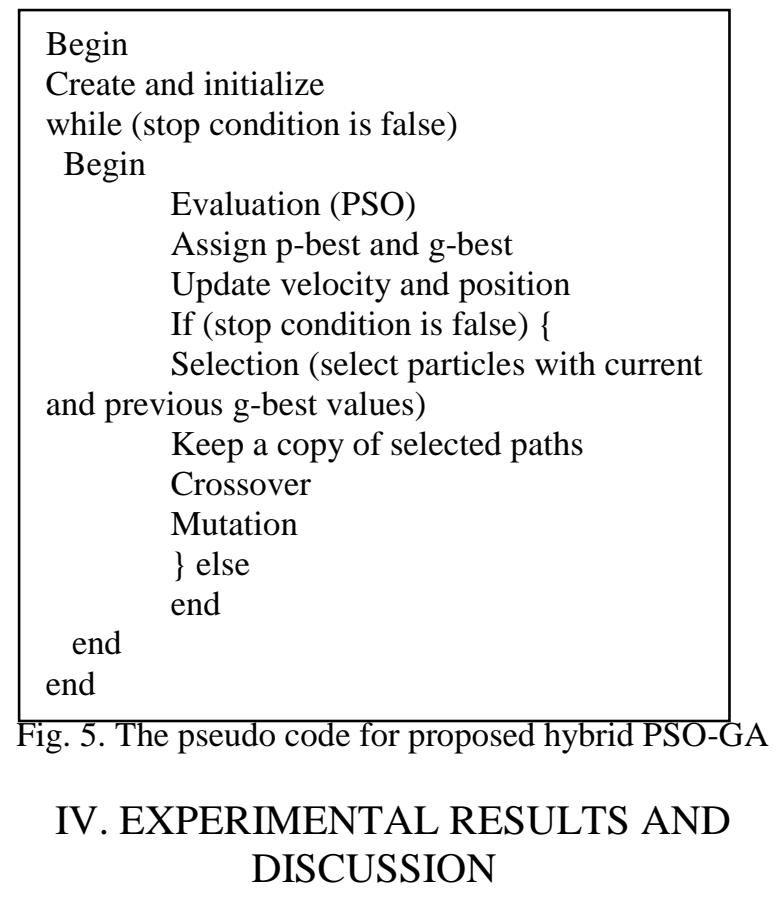

In our performed practical experiments, the proposed hybrid PSO-GA is related to and then compared with the GA and PSO algorithms. This has been achieved through computer simulations representing an Ultra Dense Network consisting of 25 nodes. All experiments were performed in the MATLAB 17 environment on Intel Core i-8 processor. The mutation probability parameter is set to 0.05 in all the 
experiments. Each run of the simulation is terminated when all the chromosomes have converged to a defined dedicated solution. Dijkstra's solution is taken as a reference and each feasible solution is compared with it to verify its accuracy and validity.

The four routing approaches that have been considered namely Dijkstra's shortest path approach, GA approach, PSO approach and our novel hybrid PSO-GA approach were implemented on different scale networks.

\section{Simulation Results for a Fixed Network with 25 Nodes:}
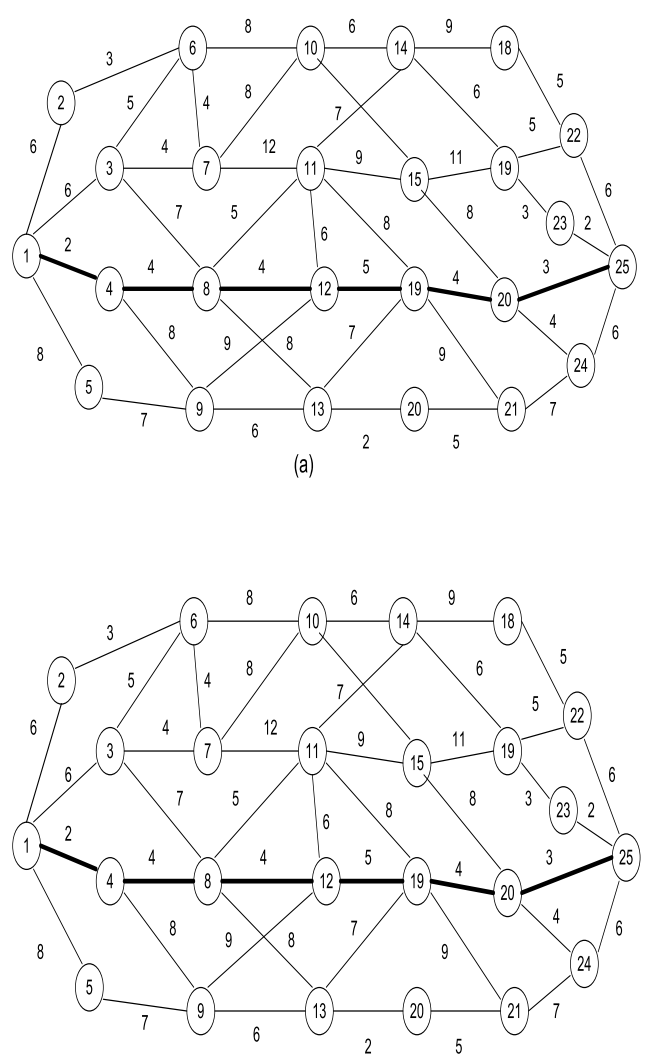

(b)

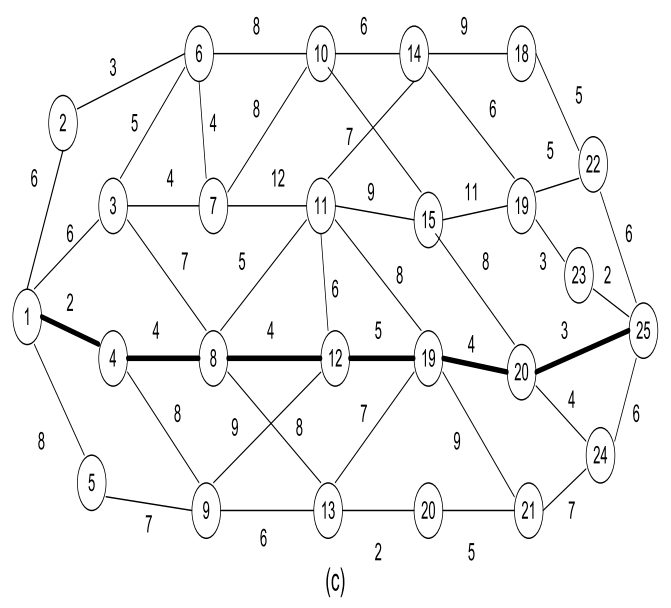

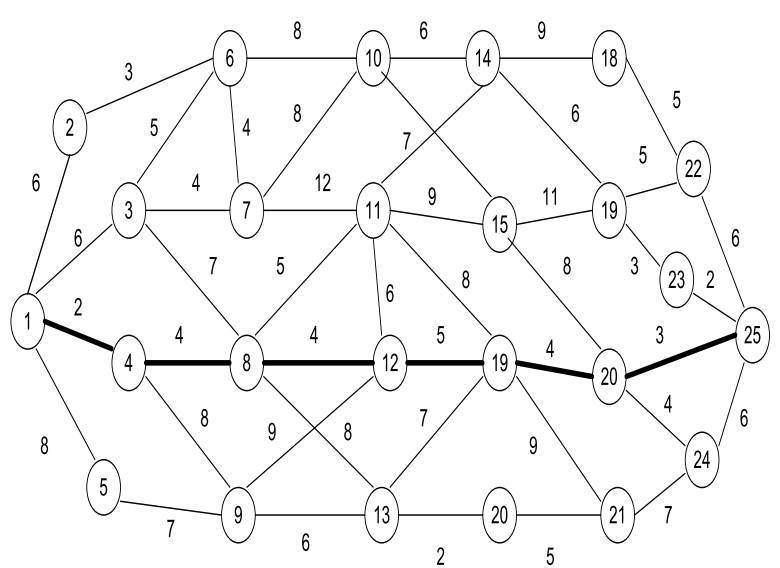

(d)

Fig.6. Display of simulation results for the paths found by each algorithm. (a) Result of the PSO algorithm (total path costs: 22). (b) Result of the Genetic algorithm (total path costs: 22). (c) Result of the hybrid PSO-GA algorithm (total path costs: 22). (d) Result of the Dijkstra's algorithm (total path costs: 22).

Initially, we have considered a 25 node Ultra Dense Network for our research and study. Subsequently, we have extended our work on different network types and scales with nodes expanding up to 150 to converge with our idea of a Heterogeneous Dense Network. A total of 500 network topologies were considered in each case. In the considered diagram, the bold line shows the optimal path (feasible solution) from the source to the destination. The size of the population is equal to the number of nodes in the network so that we can have a fair comparison of the performance and competence of the algorithms.

Fig.6 (a)-(d) shows the shortest paths found by the four algorithms among the source/destination pairs and are highlighted by bold lines. The path computed by the proposed algorithms is the same as the one computed by Dijkstra's algorithm, which is one of the most established algorithms for solving the Shortest Path Problem [15].

The above results establish the fact that all the four approaches were successful in calculating the shortest path between the source/destination pair with a total cost of 22 for a 25 node dense network. 
This has also led us to further investigate the performance of the algorithms based on different parameters and criteria.

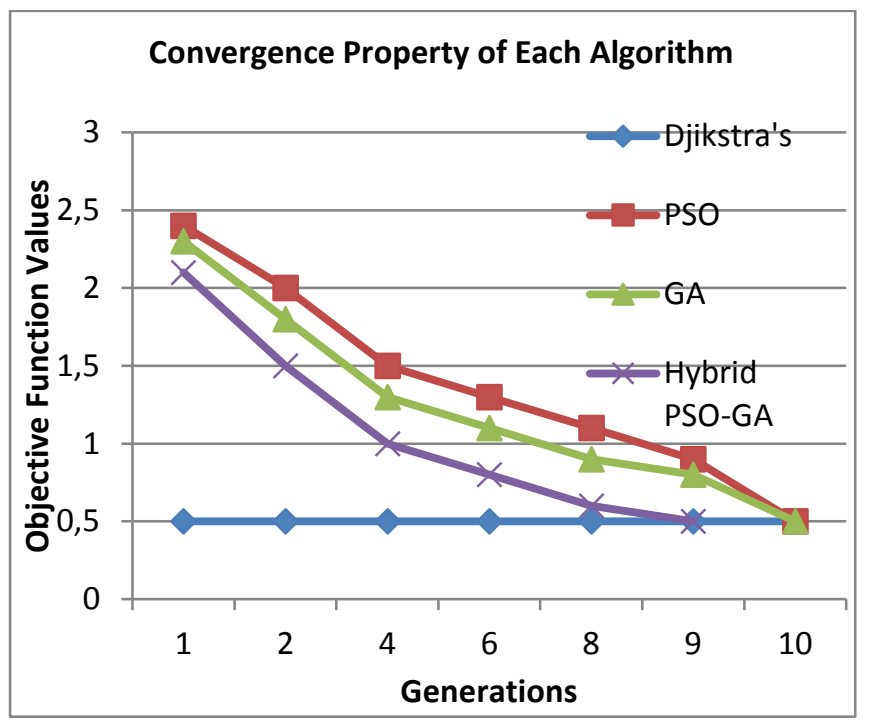

Fig.7. Convergence Property of Each Algorithm

Fig.7 displays the comparison of the objective-function values of all the four proposed algorithms against the number of iterations. From the figure, we can see that our proposed hybrid PSO-GA has the fastest rate of convergence. This is because it takes the least number of iterations to converge with Dijkstra's algorithm, whose value is always constant, whereas the other two take slightly longer number of iterations. The algorithm which overlaps with Dijkstra's algorithm in the smallest number of iterations has the best convergence property since all the algorithms have fixed size population.

\section{A. Simulation Results for Dynamic Ultra Dense Network Topologies:}

In this subsection, we try to prove that the convergence time and quality of solution of the earlier experiments holds true for all kinds of UDNs. We have extended our research and investigated UDNs of 25-150 relays nodes with source/destination pairs and normalized link costs metric.

A total of 500 network topologies were considered in each case. Firstly, we have investigated the route optimality (quality of solution) for each proposed algorithm. The route optimality is the percentage of the number of times that the proposed algorithm finds the global optimum or the shortest path of the source/destination pair. The population size of the proposed algorithms is always kept equal to the number of nodes in the networks.

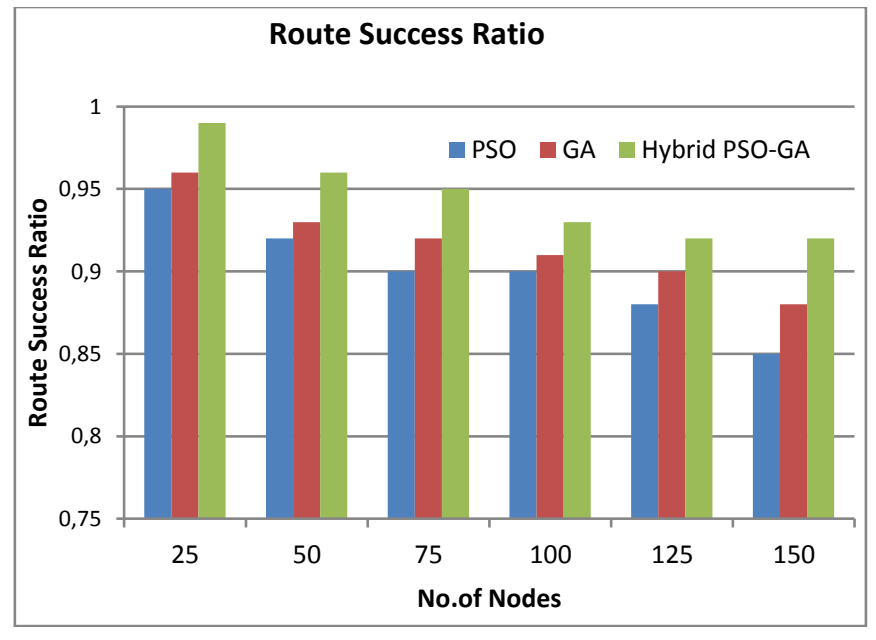

Fig.8. Comparison results of the Route Optimality for each algorithm.

Fig.8 compares the route optimality of all the proposed algorithms. From the figure, we can see that the route success ratio of our proposed hybrid PSO-GA is much higher than the other two algorithms. For instance at 25 nodes, the hybrid algorithm has a route success ratio of almost 99 percent. As the size of the network increases, the success ratio slightly dips but still outperforms the other two. At 150 nodes UDN, the hybrid PSO-GA attains a 7\% improvement over PSO and $4 \%$ improvement over the proposed GA.

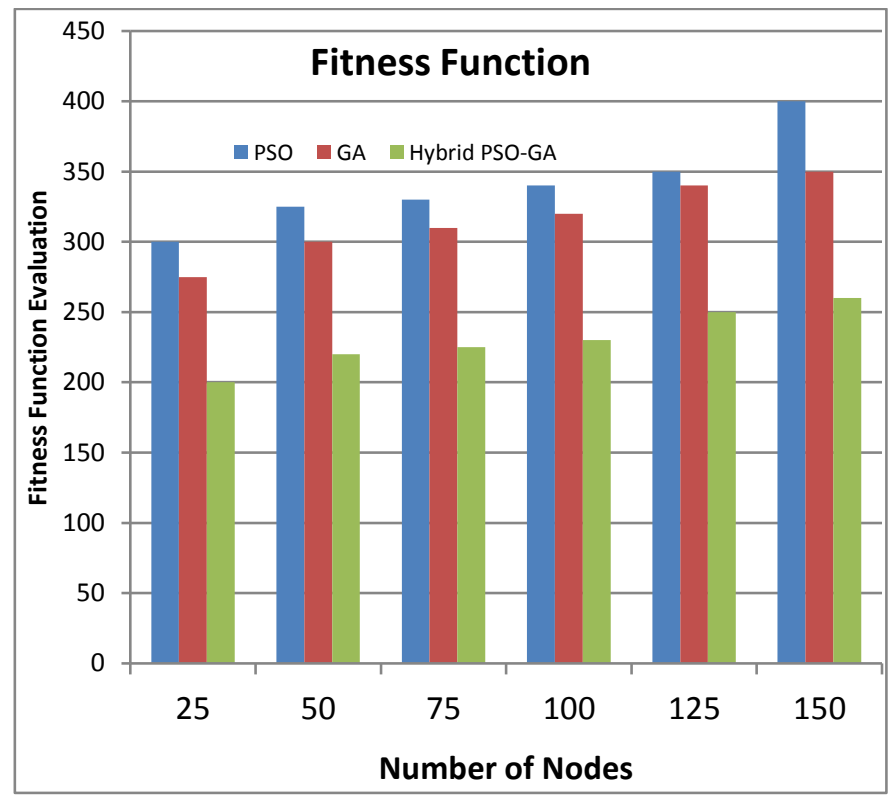

Fig.9. Comparison results of the rate of convergence for each algorithm.

Secondly, the speed of convergence of all the algorithms is investigated in terms of the average number of fitness function 
evaluations. Cant ${ }^{-} \mathrm{u}-\mathrm{Paz}$ [19] suggests that the number of fitness function evaluations (total execution time) directly measures the performance of convergence if all the algorithms converge to the defined solution with identical quality. The hybrid PSO-GA has the optimum performance since it involves the smallest number of fitness evaluations.

Fig.10 shows that the our proposed hybrid PSO-GA has much higher convergence rate because the number of fitness function evaluations needed to reach similar quality of solution is smaller than any other algorithm.

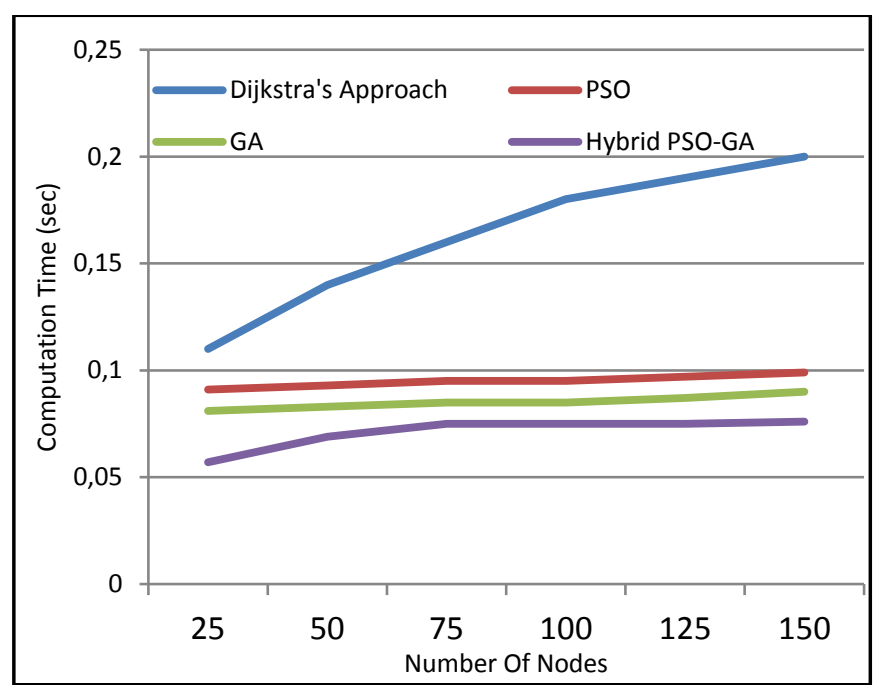

Fig.10. Convergence performance of the four algorithms in terms of computation time.

We have further measured the computation time of the four algorithms based on the earlier experiments to compare the convergence property of the hybrid PSO-GA with the rest. We have found the average computation time of Dijkstra's as 0.11 and the proposed hybrid PSO-GA as 0.057 .

Fig.10 shows that the computation time of Dijkstra's algorithm increases as the network grows from 25 to 150 nodes respectively while that of our proposed hybrid PSO-GA remains nearly constant with slight upward variations. This establishes the accuracy and efficiency of our propose work.

\section{B. Comparison of Packet Delivery Ratio (PDR), End-to-End Delay and Throughput of the three proposed approaches.}

Finally we have investigated and measured three network metrics namely Packet Delivery Ratio (PDR), End to End Delay and Throughput for the three approaches on networks of the different scales on completion of the selection of the source destination pairs. Fig.11 and Fig.12 shows that our proposed hybrid PSO-GA has delivered approximately around $25 \%$ better PDR values and $30 \%$ throughput values. Fig. 13 shows that the proposed hybrid PSO-GA method reduces the delay in data transmission approximately by about $45 \%$ compared to other two approaches.

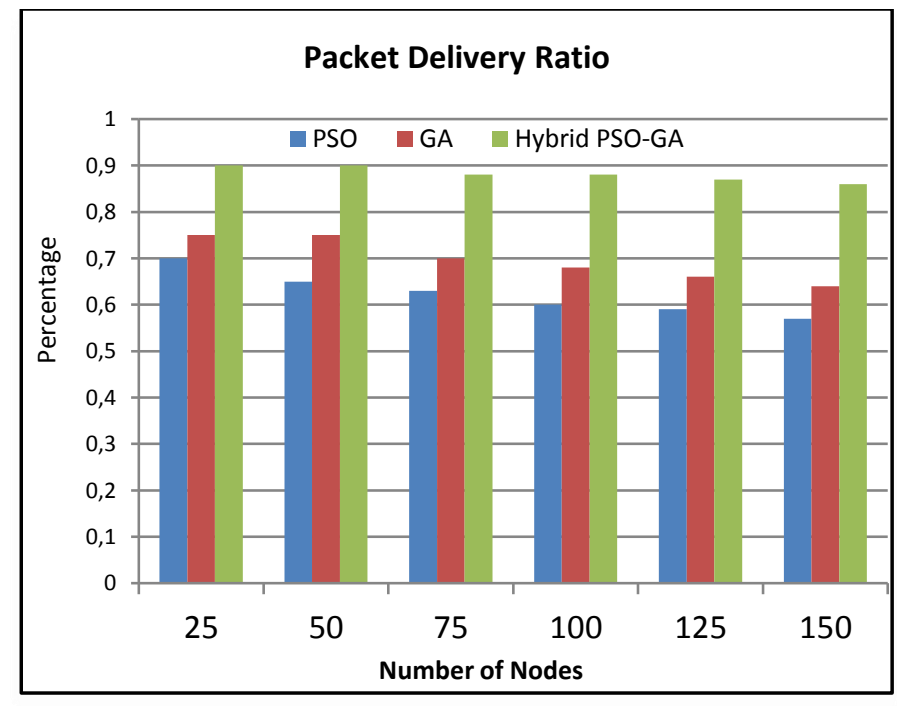

Fig.11. PDR values of the proposed approaches.

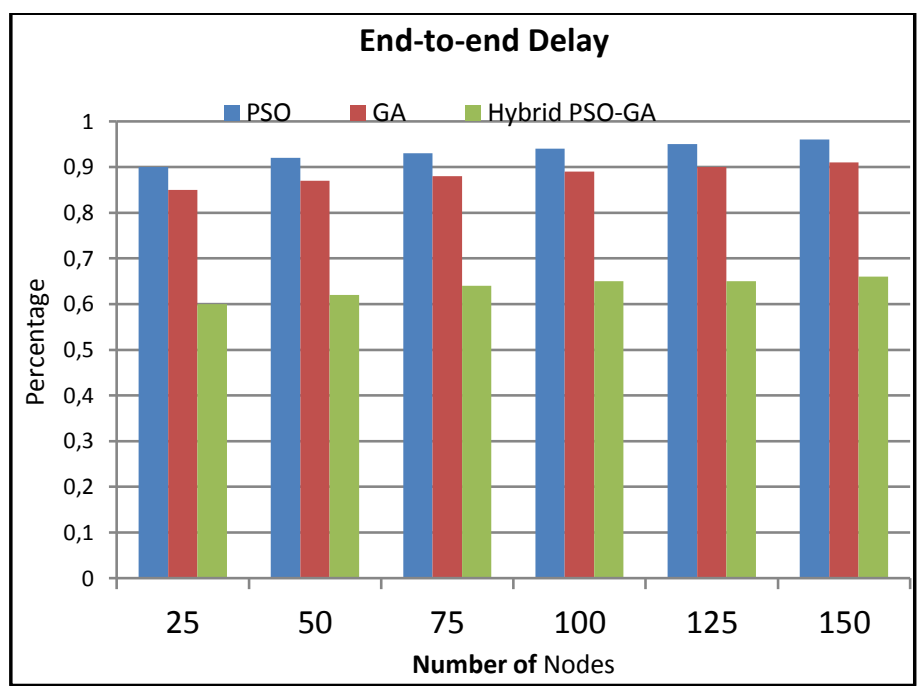

Fig.12. End to End Delay values of the proposed approaches.

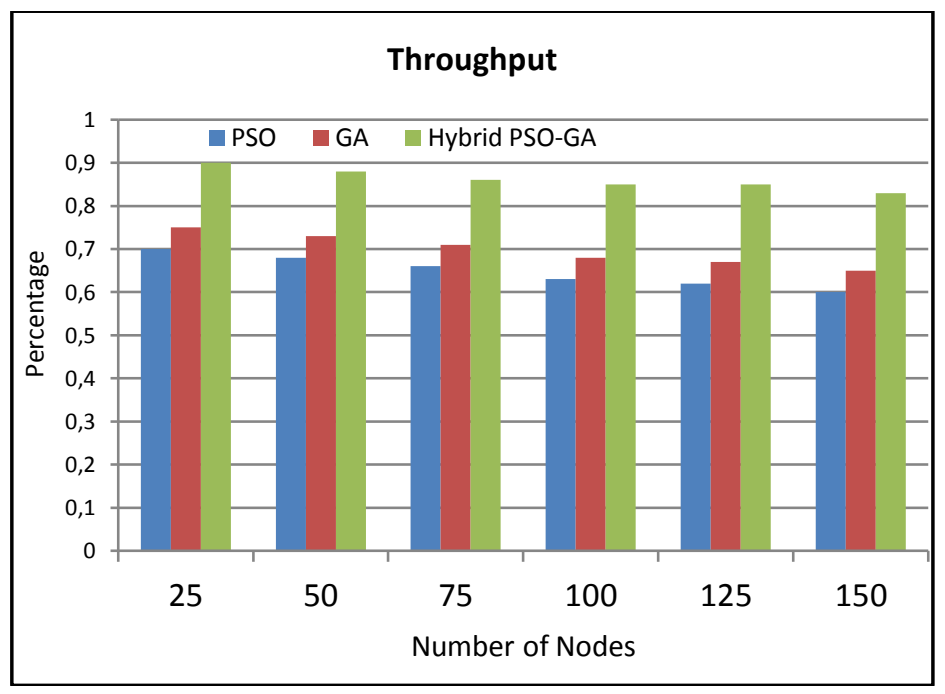

Fig.13. Throughput values of the proposed approaches. 
In 5G networks, small cell networks of varying sizes will coexist and synchronize with macro cells, either in the same spectrum or on a dedicated carrier channel. The mobility and presence of large number of users and their movements across multiple cells during an ongoing communication with make data transmission of large volumes and bandwidth a very challenging work. The normal routing procedures and algorithms will prove to be bottleneck for the realization of $5 \mathrm{G}$ goals. This implies that the optimal path between the source/destination pairs for communication and data transmission will be highly fragile and dynamic in nature. Under such alternating environments, routing procedures and algorithms to solve the Shortest Path Routing Problem (SPP) for data transfer in $5 \mathrm{G}$ networks have to be designed to be robust and scalable in nature in order to ensure seamless connectivity and quality of service (QOS) of the network.

In recent years, PSO and GA computational techniques have been applied to solve lot of optimization problems. Therefore, we have investigated and explored the performance analysis of the PSO and GA techniques through several papers [2], [3], [10][11].This investigation has given us the understanding that each one has its own advantages and disadvantages in the context of the optimization problem being applied. The problem of stagnation of PSO has also been discussed. Also the comparison between both these algorithms has been formulated in [9]. Here the author stresses on the fact that PSO performs well in the early iterations but develops problems in reaching a near-optimal solution. The reviewed literature has led us to believe that in case of our proposed work to solve the SPP problem in a 5G UDN where the size of the network increases or decreases continuously due to multiple factors, the efficiency and accuracy of the computed results using only the PSO and GA techniques can be compromised to a certain extent. Under these circumstances we have moved forward and proposed a novel hybrid PSO-GA algorithm to provide an optimal solution to the SP Routing problem in a dense network.

In the hybrid PSO-GA algorithm an effort has been made to extract and integrate the best possible features and characteristics of the both the individual algorithms. This has been reflected in the pseudo code presented earlier in the paper. This algorithm works on variable length chromosomes. Crossover and mutation operations help to maintain population diversity and protect solutions from falling into local traps. Infeasible solutions or chromosomes are also being investigated and treated using special functions without any major modifications to computing requirements. All the performed practical experiments and simulations indicate that the algorithm is insensitive to variations in network topologies in terms of speed of convergence and route optimality.

The proposed hybrid PSO-GA has the fastest rate of convergence taking 9 iterations compared to PSO and GA which takes 10 iterations to converge with Dijkstra's algorithm which is the considered standard .Also the speed of convergence of all the algorithms is investigated in terms of the average number of fitness function evaluations where also the hybrid PSO-GA has the optimum performance since it involves the smallest number of fitness function evaluations. For example, in a 75 node UDN it takes around 225 number of fitness evaluations against 325 and 310 for PSO and GA respectively. Further, convergence performance of all the algorithms is being investigated in terms of computation time. Computation time of Dijkstra's algorithm increases as the network grows from 25 to 150 nodes respectively while that of our proposed hybrid PSO-GA remains nearly constant with slight upward variations. We have found the average computation time of the proposed hybrid PSO-GA as 0.057 and that of Dijkstra's as 0.11 which establishes its supremacy over others.

We have also investigated the route optimality or quality of solution for each proposed algorithm. The route success ratio of our proposed hybrid PSO-GA is much higher than the other two algorithms. For instance at 25 nodes, the hybrid algorithm has a route success ratio of almost 99 percent and at 150 nodes UDN, the hybrid PSO-GA attains a 7\% improvement over PSO and 4\% improvement over the proposed GA.

On establishing the shortest optimal path between the source/destination pairs in terms of route optimality, the performance of the network is measured in terms of PDR, End-to-End Delay and Throughput. The proposed hybrid algorithm has delivered approximately around $25 \%$ better PDR values and $30 \%$ throughput values. Also it reduces the delay in data transmission approximately by about $45 \%$ compared to other two approaches.

\section{V.CONCLUSION}

The work carried out under this research presents three evolutionary optimization algorithms to compute and solve the Shortest Path Problem (SPP) in an 5G Ultra Dense Network (UDN).The shortest optimal path between the multiple source and destination pair was simulated for different network types and scales. Performance of the three algorithms is also investigated and evaluated based on different parameters, criteria and conditions. The performed work has led us to the conclusion that the proposed Hybrid PSO-GA has given us comparatively better solutions in nearly all the situations. In near future, we plan to include a range of other bio-inspired algorithms to further our work and study the performance of these algorithms to solve the SP problem.

\section{REFERENCES}

[1] M. Kamel, W. Hamouda and A. Youssef, "Ultra-Dense Networks: A Survey", in IEEE Communications Surveys and Tutorials, vol. 18, no. 4, pp 2522-2545, 2016.

[2] J. Kennedy and R. Eberhart, Particle Swarm Optimization, Proc. of IEEE Int. Conf. Neural Networks, vol.4, pp.1942-1948, 1995.

[3] J. Kennedy and R. Eberhart, Swarm Intelligence, Morgan Kaufmann Publishers, 2001.

[4] W. Stalling, High-Speed Networks: TCP/IP and ATM Design Principles. Englewood Cliffs, NJ: Prentice-Hall, 1998.

[5] C. W. Ahn, R. S. Ramakrishna, "A Genetic Algorithm for Shortest Path Routing Problem and the Sizing of Populations" 
IEEE transactions on evolutionary computation, vol. 6, no. 6, December 2002

[6] C. W. Ahn, R. S. Ramakrishna, C. G. Kang, and I. C. Choi, "Shortest path routing algorithm using hopfield neural network," Electron. Lett. vol. 37, no. 19, pp. 1176-1178, Sept. 2001.

[7] D. E. Goldberg, Genetic Algorithms in Search, Optimization, and Machine Learning. Reading, MA: Addison-Wesley.

[8] X. Hue, "Genetic algorithms for optimization: Background and applications, "Edinburgh Parallel Computing Centre, Univ. Edinburgh, Edinburgh, Scotland, Ver. 1.0, Feb. 1997.

[9] J. Angeline, "Evolutionary optimization versus particle swarm optimization: Philosophy and performance differences", Proc. of the 7th International Conference on Evolutionary Programming VII, pp.601-610, 1998.

[10] [10] J. Kennedy, Small worlds and mega-minds "Effects of neighborhood topology on particle swarm performance", Proc. of the 1999 Congress of Evolutionary Computation, vol.3, pp.1931-1938, 1999.

[11] J. A. Vasconcelos, J. A. Ramirez, R. H. C. Takahashi, and R. R. Saldanha "Improvements in Genetic Algorithms" IEEE Transactions On Magnetics, Vol. 37, No. 5, September 2001

[12] D. López-Pérez, M. Ding, H. Claussen, and A. H. Jafari, "Towards $1 \mathrm{Gbps} / \mathrm{UE}$ in cellular systems: Understanding ultradense small cell deployments," IEEE Communication. Surveys Tutorials, vol. 17, no. 4,pp. 2078-2101, 4th Quart., 2015.

[13] M. Ding, D. López-Pérez, G. Mao, P. Wang, and Z. Lin, "Will the area spectral efficiency monotonically grow as small cells go dense" in Proc. IEEE Glob. Communication. Conf. (GLOBECOM), San Diego, CA, USA, Dec. 2015, pp. 1-7.

[14] J. Deissner and G. P. Fettweis, "A study on hierarchical cellular structures with inter-layer reuse in an enhanced GSM radio network," in Proc. IEEE Int. Workshop Mobile Multimedia Communication. (MoMuC), San Diego, CA, USA, 1999, pp. 243-251.

[15] DIJKSTRA EW. 1959. A note on two problems in connexion with graphs. Numerische mathematik, 1(1): 269-271.

[16] X. Ge, S. Tu, G. Mao, C. Wang and T. Han, "5G Ultra- Dense Cellular Networks", in IEEE Wireless Communications, vol.23, no. 1, pp. $72-79,2016$.

[17] J. Zhang, D. Zhang, K. Xie, S. He1, H. Qiao and B. Zeng "A Cooperative Routing Algorithm for Maximizing Network Lifetime", Springer-Verlag Berlin Heidelberg 2013, R. Wang and F. Xiao (Eds.): CWSN 2012, CCIS 334, pp. 665-6752013.

[18] A. Mohemmed, N.C.Sahoo, T.K.Geok "Solving shortest path problem using particle swarm optimization" Applied Soft Computing .8.1643-1653. 10.1016/j.asoc.2008.01.002.

[19] E. Cant u-Paz," Efficient and Accurate Parallel Genetic Algorithms." Norwell, MA: Kluwer, 2000.

\section{Creative Commons Attribution License 4.0 (Attribution 4.0 International, CC BY 4.0)}

This article is published under the terms of the Creative Commons Attribution License 4.0

https://creativecommons.org/licenses/by/4.0/deed.en US 